\title{
Structural Mechanism of Inhibition of the Rho Transcription Termination Factor by the Antibiotic Bicyclomycin
}

\author{
Emmanuel Skordalakes, ${ }^{1}$ Andrew P. Brogan, ${ }^{2}$ \\ Boon Saeng Park, ${ }^{2}$ Harold Kohn, ${ }^{2}$ \\ and James M. Berger ${ }^{1, *}$ \\ ${ }^{1}$ Department of Molecular and Cell Biology \\ University of California, Berkeley \\ 237 Hildebrand Hall 3206 \\ Berkeley, California 94720 \\ ${ }^{2}$ Division of Medicinal Chemistry and Natural Products \\ School of Pharmacy \\ University of North Carolina at Chapel Hill \\ 328A Beard Hall, CB 7360 \\ Chapel Hill, North Carolina 27599
}

\section{Summary}

Rho is a hexameric RNA/DNA helicase/translocase that terminates transcription of select genes in bacteria. The naturally occurring antibiotic, bicyclomycin (BCM), acts as a noncompetitive inhibitor of ATP turnover to disrupt this process. We have determined three independent X-ray crystal structures of Rho complexed with BCM and two semisynthetic derivatives, 5a-(3formylphenylsulfanyl)-dihydrobicyclomycin (FPDB) and 5a-formylbicyclomycin (FB) to 3.15, 3.05, and $3.15 \AA$ A resolution, respectively. The structures show that $\mathrm{BCM}$ and its derivatives are nonnucleotide inhibitors that interact with Rho at a pocket adjacent to the ATP and RNA binding sites in the C-terminal half of the protein. BCM association prevents ATP turnover by an unexpected mechanism, occluding the binding of the nucleophilic water molecule required for ATP hydrolysis. Our data explain why only certain elements of $\mathrm{BCM}$ have been amenable to modification and serve as a template for the design of new inhibitors.

\section{Introduction}

Rho is a hexameric RNA/DNA helicase/translocase that actively terminates transcription to regulate the expression of approximately half of the open reading frames in bacteria (Gogol et al., 1991; Oda and Takanami, 1972; Richardson, 2002; Yu et al., 2000; Zhu and von Hippel, 1998). To carry out this function, Rho initially binds to a region of C-rich mRNA (known as a rut site) that is usually found upstream from the start of specific genes or at the ends of genes (Richardson, 2002). Recognition of rut sites is accomplished by the $\mathrm{N}$-terminal third of Rho, a region termed the primary RNA binding site (Alifano et al., 1991; Morgan et al., 1985). Once bound, Rho engages the message downstream of the rut site through the use of secondary RNA binding sites that reside in its $C$ terminus (Xu et al., 2002). The C-terminal domain carries out ATP binding and hydrolysis, which powers Rho along the mRNA and assists RNA/DNA heteroduplex unwinding. Through these combined functions,
Rho is able to terminate transcription by disengaging the newly synthesized message from the template DNA and a stalled RNA polymerase.

Phylogenetic and structural data have shown that Rho is a member of the RecA superfamily of ATPases and a close homolog of $F_{1}$-ATPase (Miwa et al., 1995; Yu et al., 2000). The active Rho assembly has six ATP binding pockets located at each of the interfaces between the C-terminal domains of adjacent protomers. The ATP binding site is formed by two signature elements known as the Walker-A ( $P$ loop) and Walker-B motifs. Two other conserved sequence motifs, the $Q$ and $R$ loops, lie in close proximity to the ATP binding pocket, forming the secondary RNA binding sites of Rho. Of these, the $R$ loop is positioned in a location analogous to the motif III region of helicase superfamily proteins (Gorbalenya et al., 1989; Hall and Matson, 1999) and is thought to act as an allosteric effector switch that couples ATP turnover to RNA movement through the hole of the ring (Gogol et al., 1991; Richardson, 2002; Vincent et al., 2000a; Xu et al., 2003, 2002; Yu et al., 2000).

The active conformation of Rho, like other hexameric helicases and translocases, is thought to be that of a closed ring (Patel and Picha, 2000). The threading of an extended nucleic acid chain through a toroid is a topological challenge that is often overcome through the use of specialized mechanisms. Based on structural evidence, the loading of Rho onto RNAs is thought to proceed by a ring opening mechanism that splays two adjacent protomers apart to allow the entry of ssRNA in the interior of the particle (Gogol et al., 1991; Oda and Takanami, 1972; Skordalakes and Berger, 2003; Yu et al., 2000). Crystallographic studies have shown that Rho binds ATP in its open ring state but requires an intersubunit rearrangement to adopt a structure competent to hydrolyze ATP (Skordalakes and Berger, 2003). Interaction of ssRNA with the secondary RNA binding sites activates ATP hydrolysis and is thought to be a key step leading to ring closure (Brennan et al., 1987; Burgess and Richardson, 2001; Kim and Patel, 2001; Lowery-Goldhammer and Richardson, 1974; Stitt, 2001; Walstrom et al., 1997).

Rho is unique to bacteria and essential for the viability of many bacterial species. As a consequence, Rho is an attractive target for drug development. The natural product bicyclomycin (BCM), first isolated in 1972 from Streptomyces sapporonensis (Kamiya et al., 1972) and Streptomyces aizumenses (Miyamura et al., 1972, 1973), has been shown to specifically target Rho (Figure 1) (Zwiefka et al., 1993). BCM is a heterocyclic compound that is biosynthetically derived from L-leucine and L-isoleucine (Iseki et al., 1980; Miyoshi et al., 1980) and has antibacterial activity against gram-negative bacteria (Kamiya et al., 1972; Miyamura et al., 1972, 1973) and the gram-positive bacterium Micrococcus luteus (Nowatzke et al., 1997). BCM is commercially available under the name Bicozamycin and has been used for the treatment of nonspecific diarrhea in animals (Ericsson et al., 1985; Uemura et al., 2003). 

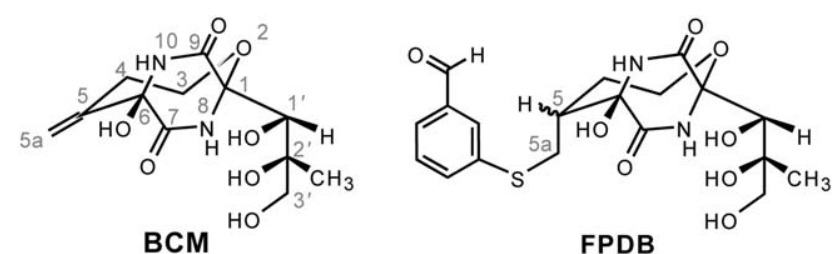

FPDB

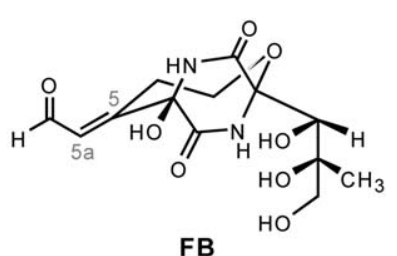

FB
Figure 1. Chemical Structures of Bicyclomycin-Based Compounds (A) Structure of the naturally occurring antibiotic bicyclomycin (BCM). (B) Structure of the analog FPDB.

(C) Structure of the analog FB.

Affinity labeling and biochemical studies have suggested that BCM exerts its activity by binding at the interface of adjacent C-terminal domains of Rho (Brogan et al., 2003; Magyar et al., 1999; Riba et al., 1998; Vincent et al., 2000a). Specifically, the BCM binding site has been proposed to lie adjacent to but distinct from the ATP site and is thought to partially overlap with the secondary RNA binding sites. Consistent with this hypothesis, kinetic studies have shown BCM to be a reversible, noncompetitive inhibitor of ATP turnover (Park et al., 1995). BCM also slows Rho's movement along RNA and acts as a mixed-type inhibitor for RNA binding at the secondary site (Magyar et al., 1996). Thus, BCM may disrupt RNA tracking directly by perturbing the secondary RNA binding sites at the same time that it interferes with Rho's ATPase activity (Carrano et al., 1998; Magyar et al., 1996; Park et al., 1995); alternatively, the effects of BCM may arise because ATP binding and hydrolysis are allosterically coupled to RNA engagement. In the cell, BCM attenuates the ability of Rho to reach and dissociate the RNA polymerase from its DNA template, yielding unnaturally long RNA transcripts (Magyar et al., 1996; Zwiefka et al., 1993). The inhibitory properties of BCM are unique among known helicase/translocase inhibitors, nearly all of which are competitive nucleotide analogs or mimics of the ATP hydrolysis transition state.

Although efficacious, the potency of BCM is tempered by an $I_{50}$ in the low micromolar range (Magyar et al., 1996, 1999; Park et al., 1995; Zwiefka et al., 1993). In attempts to improve inhibitory activity and generate more effective antibiotics, BCM has been subjected to extensive chemical modifications (Park $\mathrm{Hg}$ et al., 1996a, 1996b; Santillan et al., 1996, 1998; Vincent et al., 2001). To a large extent, most modifications either partially or completely abrogate the ability of the semisynthetic derivative to inhibit Rho. However, select modifications at the $C(5)-C(5 a)$ exomethylene have led to compounds with comparable or better inhibitory activity compared to the parent compound (Park Hg et al., 1996b; Vincent et al., 2001). Two derivatives in particular, 5a-(3-formylphenylsulfanyl)-dihydrobicyclomycin (FPDB; Figure 1B) and 5a-formylbicyclomycin (Vincent et al., 2000b) (FB; Figure 1C), show a $\sim 2$ - to 15-fold improvement in Rho inhibitory activity. Upon treatment with $\mathrm{NaBH}_{4}$, both of these compounds irreversibly modify Rho. For FPDB, covalent modification occurs at Lys181, while FB adduction proceeds at Lys336 (Vincent et al., 2000a).

Despite extensive mutagenesis, biochemical, and kinetic studies, the detailed mechanism by which BCM inhibits Rho remains unclear. While a model for the BCM binding pocket in Rho has been generated using the $F_{1}$ ATPase (Magyar et al., 1999; Moyse et al., 2000; Vincent et al., 2000a, 2000b), the exact location of this region, the orientation of the drug within the binding pocket, and the type of contacts that occur between the protein and the antibiotic are unknown. To address these issues, we solved the structure of full-length $E$. coli Rho in complex with BCM, FPDB, and FB. Our data show that the antibiotic binds at a pocket located at the interface between adjacent C-terminal domains that is formed in part by the P and R loops. Unexpectedly, $\mathrm{BCM}$ association displaces the catalytic water molecule required for ATP hydrolysis (Abrahams et al., 1994; Braig et al., 2000; Weber et al., 2000). Taken together, the structures of the Rho•bicyclomycin complexes reveal a new mode of ATPase inhibition by a small molecule agent, explain why BCM has been difficult to modify, and set the stage for future efforts to develop more effective bicyclomycin analogs.

\section{Results}

In a recent study, we determined the structure of inhibitor-free Rho bound to AMPPNP and RNA (Skordalakes and Berger, 2003). Using this effort as a starting point, we cocrystallized and determined three independent

\begin{tabular}{llll}
\hline Table 1. Data Collection & & & \\
\hline & BCM & FPDB & FB \\
\hline Space group & C2 & C2 & C2 \\
Unit cell dimensions (Å) & $211.9,111.4,161.0 ; 108.8$ & $119.5,206.9,148.6 ; 97.0$ & $210.5,109.7,160.0 ; 108.2$ \\
Resolution (A) & $50-3.15$ & $50-3.05$ & $50-3.15$ \\
Redundancy & 1.9 & 1.9 & 1.9 \\
Completeness (\%) & $88(86)$ & $92(81)$ & $86(81)$ \\
$\mathrm{R}_{\text {sym }(\%)^{\mathrm{a}}}$ & $6.5(45)$ & $5.1(54)$ & $6.1(41)$ \\
$\mathrm{I} \sigma(\mathrm{I})$ & $7.5(2.0)$ & $11.0(2.0)$ & $9(2.1)$ \\
\hline
\end{tabular}

Values in parentheses are for the highest-resolution bin.

${ }^{a} R_{\text {sym }}=\Sigma\left(\mid I_{h k l}-\left\langle l_{h k l}>\right|\right) / \Sigma\left(I_{h k l}\right)$, where $I_{h k l}$ represents intensities for symmetry-related reflections. 


\begin{tabular}{|c|c|c|c|}
\hline & $\mathrm{BCM}$ & FPDB & FB \\
\hline Resolution (Å) & $20-3.15$ & $20-3.05$ & $20-3.15$ \\
\hline $\mathrm{R}_{\text {work }}(\%)^{\mathrm{a}}$ & 26.5 & 26.3 & 26.0 \\
\hline$R_{\text {free }}(\%)^{b}$ & 30.5 & 29.5 & 29.6 \\
\hline Rmsd bonds ( $(\AA)$ & 0.008 & 0.006 & 0.006 \\
\hline Rmsd angles $\left({ }^{\circ}\right)$ & 1.35 & 1.07 & 1.08 \\
\hline \multicolumn{4}{|l|}{ Average B factors $\left(\AA^{2}\right)$} \\
\hline Protein & 58.7 & 70.3 & 56.2 \\
\hline Water & N/A & 148 & N/A \\
\hline Bicyclomycin & 56 & 62 & 59 \\
\hline \multicolumn{4}{|l|}{ Number of atoms } \\
\hline Protein & 19,086 & 19,086 & 19,086 \\
\hline Water & 0 & 20 & 0 \\
\hline $\mathrm{BCM}$ & 21 & 30 & 23 \\
\hline \multicolumn{4}{|c|}{ Ramachandran analysis (\%) } \\
\hline Most favored & 84.5 & 86.8 & 85.2 \\
\hline Additional allowed & 14.4 & 12.3 & 13.8 \\
\hline Generously allowed & 1.1 & 0.9 & 1.0 \\
\hline
\end{tabular}

${ }^{a} R_{\text {work }}=\Sigma\left(\mid F_{\text {obs }}-F_{\text {calc }}\right) / \Sigma\left(F_{\text {obs }}\right)$, where $F_{\text {obs }}$ represents observed structure-factor amplitudes, and $F_{\text {calc }}$ represents calculated structurefactor amplitudes.

${ }^{b} R_{\text {free }}$ is the $R$ value calculated for a test set of reflections comprising a randomly selected $5 \%$ of the data that is not used during refinement.

structures of full-length $E$. coli Rho in the presence of $\mathrm{Mg}^{2+}, \mathrm{ATP} \gamma \mathrm{S}$, an $\mathrm{r}(\mathrm{CU})_{4}$ oligonucleotide, and either BCM, FPDB, or FB at 3.15, 3.05, and $3.15 \AA$ resolution (Tables 1 and 2). In all instances, the protein-inhibitor complexes cocrystallized in the monoclinic space group C2. Rho•BCM and Rho•FB crystals have increased unit cell dimensions compared to the inhibitor-free crystal form of Rho, while Rho•FPDB crystals exhibit dimensions very similar to the drug-free form. In all three cases, there is one intact hexamer, and thus six independent views, of each monomer in the asymmetric unit. The structures of each of the inhibitor-bound forms were solved by molecular replacement using the original inhibitor- and AMPPNP-free structure of Rho (1PV4) after omitting the coordinates for the RNA.

\section{Rho Structure}

Each Rho protomer consists of two distinct domains connected by an extended linker. The $\mathrm{N}$-terminal domain consists of three $\alpha$ helices appended to a fivestranded $\beta$-barrel comprising an oligonucleotide/oligosaccharide binding OB fold (Cavarelli et al., 1993; Schindelin et al., 1994). The OB fold binds a cytosine dinucleotide and represents the primary RNA binding site of Rho (Allison et al., 1998; Martinez et al., 1996a, 1996b). The C-terminal domain is a RecA-type ATP binding fold that consists of seven parallel $\beta$ strands sandwiched between four $\alpha$ helices (Abrahams et al., 1994; Story et al., 1992; Toth et al., 2003). This region also contains the secondary RNA binding sites formed by the $Q$ and $R$ loop signature sequence motifs (Miwa et al., 1995; Skordalakes and Berger, 2003).

In each of our crystal structures, six protomers assemble into an open hexameric ring that is thought to represent a state of the molecule capable of loading onto mRNA (Skordalakes and Berger, 2003) (Figure 2A). In all three structures (Rho•BCM, Rho•FPDB, and Rho $\cdot \mathrm{FB}$ ), there is clear density for residues $1-417$ of all six protomers. Comparison of the BCM- and FB-bound structures with the inhibitor-free form of Rho shows that a modest degree of global structural change oc- curs upon drug binding ( $\alpha \alpha$ rmsd $2.8 \AA$ ). These differences are equally distributed throughout the ring and lead to a slight contraction of the hexamer. As a consequence, the "A" and " $F$ " protomers at the opening of the ring are offset from each other by $40 \AA$, as compared to $45 \AA$ for the inhibitor-free complex. Concomitantly, the gap between these protomers narrows from 12 to $8 \AA$. The inhibitor-induced conformational change observed here may be associated with the binding of the inhibitor near the secondary RNA binding site ( $R$ loop), which upon RNA binding is thought to drive ring closure (Engel and Richardson, 1984; Kim et al., 1999; Shigesada and Wu, 1980). By contrast, the Rho-FPDB structure is nearly identical to the drug-free state $(\mathrm{C} \alpha$ rmsd of $0.51 \AA$ ).

\section{ATP Binding}

The ATP binding pocket of Rho is located at the interface between the $\mathrm{C}$-terminal domains of adjacent protomers and is formed in part by residues from the Walker-A and -B motifs (Figure 2). There is clear electron density for ATP $\gamma \mathrm{S}$ in all six ATP binding pockets (Figure 2C). The adenosine base and the $\alpha$ - and $\beta$-phosphates of the nucleotide make contacts typical of those seen for other oligomeric RecA-type proteins. However, while the triphosphate moiety of each nucleotide is cradled by the backbone amides of the $\mathrm{P}$ loop, interactions between the $\gamma$-phosphate and other key elements of the protein are not canonical for a catalytically active ATPase. Most notably, as observed in the original Rho crystal structure, the opening of the ring splays apart adjacent subunits, widening the ATP binding site and reconfiguring it into an inactive conformation (Skordalakes and Berger, 2003). The use of subunit displacements and rotations to alter the architecture of the ATP binding pocket of oligomeric RecA-type ATPases also has been observed in related helicases and strand exchange proteins, and probably reflects a degree of overlap in the mechanisms used by these proteins to open the catalytic cleft following hydrolysis, expel $A D P \bullet P_{i}$, and prime the site to accept an incoming nu- 


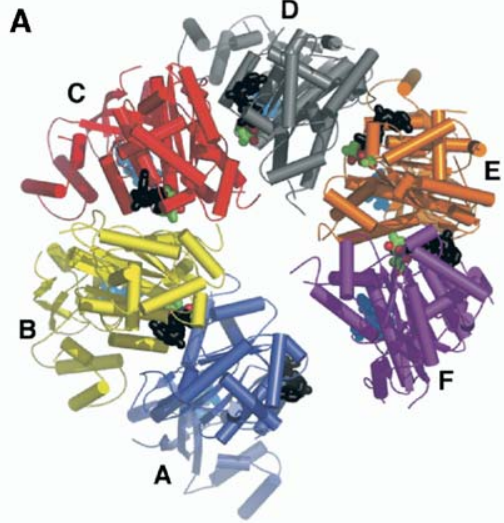

B

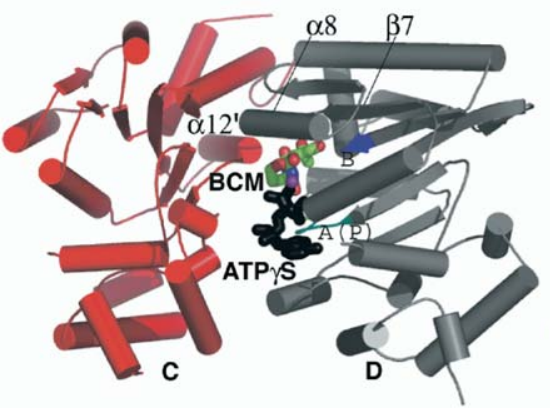

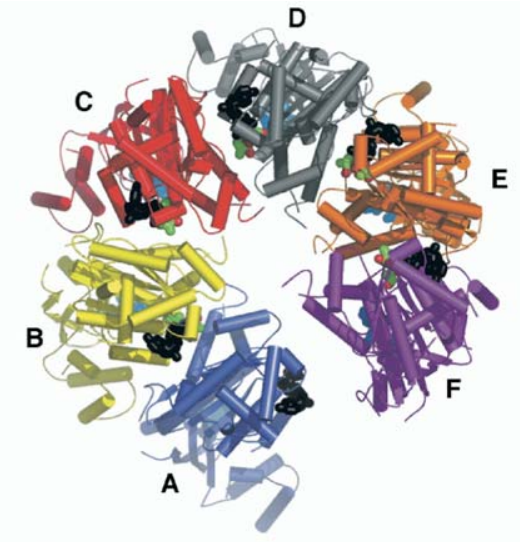

C

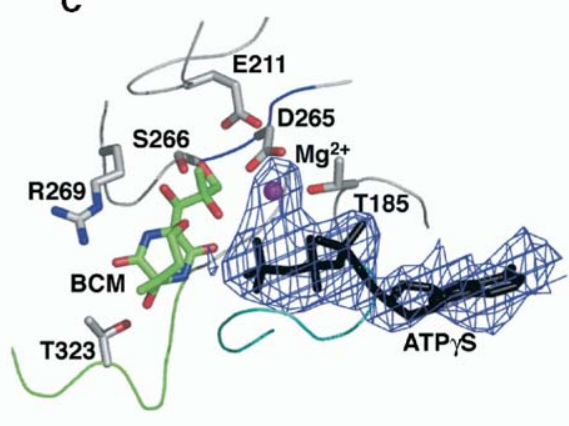

Figure 2. Structure of the Rho•r(CU) ${ }_{4} \bullet A T P \gamma S \bullet B C M$ Complex

(A) Stereo representation of the hexameric open-ring Rho structure looking down the pseudo six-fold axis of the C-terminal face of the molecule. Each protomer is colored differently. RNA, ATP $\gamma \mathrm{S}$, and BCM are shown as blue, black, and green/red sticks, respectively.

(B) Dimer interface of two C-terminal domains (protomers C and D) showing the location of Rho-bound BCM (green sticks) with respect to the ATP binding pocket. The signature sequence Walker-A and -B motifs are labeled and shown in cyan and blue, respectively. The perspective looks from outside the edge of the ring toward the interior.

(C) $3 \sigma F_{o}-F_{c}$ electron density for ATP $\gamma \cdot \mathrm{Mg}^{2+}$. The antibiotic, ATP $\gamma$, and the residues of Rho involved in contacts with bicyclomycins are shown in stick representation.

cleoside-triphosphate (Conway et al., 2004; Sawaya et al., 1999; Singleton et al., 2000; Story et al., 1992; VanLoock et al., 2003; Wu et al., 2004)

One significant difference we observe between the inhibitor-bound structures of Rho and the original inhibitor-free model is that $\mathrm{Mgg}^{2+}$ ion now clearly occupies its canonical position within each ATP binding site (Figure $2 \mathrm{C}$ ). The divalent metal is liganded by the $\beta$ - and $\gamma$-phosphates of the bound nucleotide and by T185 of the Walker-A motif. The $\mathrm{Mg}^{2+}$ ion also contacts the $\mathrm{C}\left(2^{\prime}\right) \mathrm{OH}$ of the bicyclomycins; this interaction completes the octahedral coordination geometry of the metal and appears to stabilize $\mathrm{Mg}^{2+}$ binding.

\section{Bicyclomycin Binding}

Despite the modest resolution of our diffraction data for the Rho•bicyclomycin cocrystal structures (3.05-3.15 Å), phases from molecular replacement solutions using an inhibitor-free model of Rho (1PV4) showed clearly interpretable electron density for bicyclomycin binding to the protein (Figure 3 and Supplemental Figure S1). Density is evident in all possible binding sites except for protomer A, which lies at the opening of the ring. Like ATP, the inhibitors occupy a pocket formed at the interface between adjacent C-terminal domains (Figures 2B and $3 \mathrm{D})$. The bicyclomycin binding site is composed of segments from the $P$ loop, $R$ loop, and $\beta 7 / \alpha 8$ connector loop of one protomer, as well as helix $\alpha 12^{\prime}$ of the adjacent protomer (for clarity, residues and structural elements provided by the protomer that abuts the principle drug binding site of a neighboring subunit will be designated with a prime) (Figure $2 \mathrm{~B}$ ). The pocket is approximately $12 \AA$ deep and $10 \AA$ wide and is lined by the side chains of residues Glu211, Arg212, Asp265, Ser266, and Arg269 of one protomer and by the $\mathrm{C} \alpha$ of Gly337' of the adjacent protomer. Each of the bicyclomycin compounds also interacts with the nucleotide and its associated $\mathrm{Mg}^{2+}$ ion. The electron density is of sufficient quality to distinguish BCM, FPDB, and FB in all three cocrystals (Figure 3 and Supplemental Figure S1).

The [4.2.2] piperazinedione ring and $C(1)$ triol group form the main structural components of BCM (Figure 1). This region docks snugly into its binding pocket and forms numerous contacts with Rho (Figures 3D, 4A, and 4B; Table 3). The $C(1)$ triol group extends into the closed end of the pocket, where its hydroxyl groups at the $\mathrm{C}\left(2^{\prime}\right)$ and $\mathrm{C}\left(3^{\prime}\right)$ positions create a network of hydrogen bonds with $O \in 2$ (Protein Data Bank [PDB] numbering scheme) of Glu211, $N \in 1$ of Arg212, $0 \delta 1$ of Asp265, O $\gamma$ of Ser266, $\mathrm{N} \in 2$ of Arg269, and the $\mathrm{Mg}^{2+}$ ion. The [4.2.2] piperazinedione ring system spans the widest diameter of the pocket and positions the amide car- 

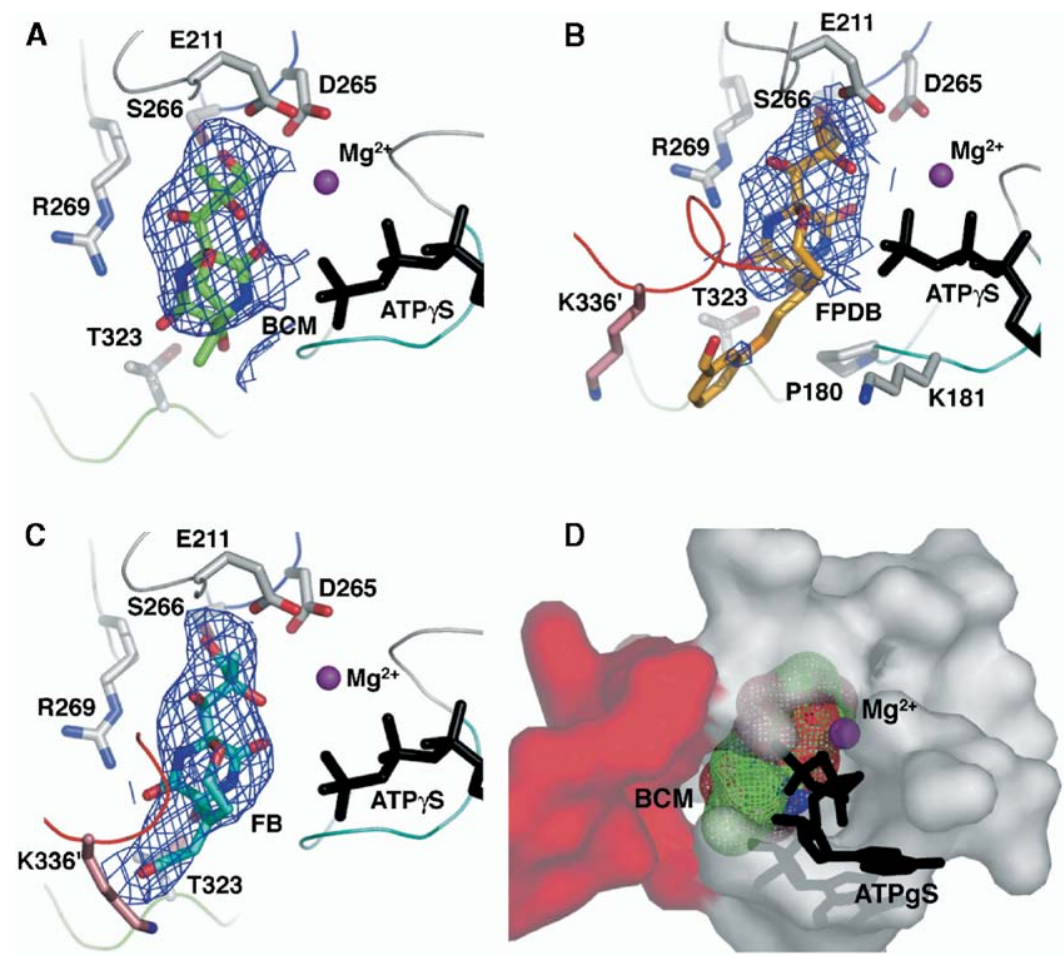

Figure 3. Electron Density for Inhibitor Complexes

The inhibitor binding site is shown with experimental, multidomain-averaged electron density (1.5 $\sigma$ contour level) around each compound. Residues of Rho involved in contacts with bicyclomycins are shown in stick representation. (A), BCM; (B), FPDB; (C), FB. (D) shows a surface representation of the Rho-dimer interface showing the volume occupied by BCM. The two protomers are positioned and colored similarly as in Figure 2B. The antibiotic is shown with a green/red mesh surface, while ATP $\gamma \mathrm{S}$ is shown as black sticks.

bonyls within hydrogen bonding distance of $\mathrm{N} \in 2$ of Arg269 on one side and one of the nonbridging oxygens of the $\gamma$-phosphate of ATP $\gamma S$ on the other. The aliphatic portion of the ether moiety of [4.2.2] piperazinedione ring lies equidistant from the $\gamma$-phosphate of ATP $\gamma \mathrm{S}$ of one protomer and Gly337' of the other, while its oxygen hydrogen bonds to $\mathrm{N} \in 1$ of Arg212.

The interactions between Rho and both FPDB and FB are similar to those seen for BCM but exhibit some compound-specific differences. In the case of FPDB, the thiobenzaldehyde group attached to position $\mathrm{C}(5 \mathrm{a})$ of the [4.2.2] piperazinedione ring extends out of the BCM binding pocket and wedges itself between the hydrophobic side chain of Pro180 of one protomer and the aliphatic portion of the side chain of Lys336' from the adjacent protomer (Figures 3B and 4B). The aldehyde moiety of the thiobenzyldehyde group is also within hydrogen bonding distance from the $\mathrm{N} \zeta$ of Lys181. Although the high temperature factors and broken electron density for both the Lys 181 side chain and the thiobenzaldehyde group indicate that this region is poorly ordered, their juxtaposition is consistent with $\mathrm{NaBH}_{4}$ crosslinking data (Vincent et al., 2000a). In the case of FB, which contains a formyl group appended to the $C(5 a)$ double bond, the inhibitor makes only one additional interaction with Rho, hydrogen bonding directly to Lys336' of the protomer adjacent to the ATP binding site (Figures $3 \mathrm{C}$ and $4 \mathrm{~B}$ ). This configuration likewise agrees with prior crosslinking studies (Riba et al., 1998).

\section{Discussion}

Bicyclomycin is the only known selective, clinically significant inhibitor of Rho. It is also one of the few nonnucleotide agents capable of inhibiting an oligomeric RecA-superfamily ATPase. The cocrystal structures of Rho with the naturally occurring antibiotic and the two semisynthetic bicyclomycins, FPDB and FB, explain several important aspects of inhibitor binding and function. With these data in hand, we can now understand the molecular basis for BCM inhibition and resistance and can explain why attempts to improve $\mathrm{BCM}$ binding by chemically modifying the naturally occurring antibiotic have been largely unsuccessful.

\section{BCM Resistance}

To date, numerous mutants of Rho that confer BCM resistance have been isolated. All of these are located between residues Thr158 and Leu345 in the C-terminal domain (Magyar et al., 1999; Moyse et al., 2000; Zwiefka et al., 1993) (Figure 5A). These mutations include substitutions at Ser266, Gly337, Met219, and Leu208. All of these lie within or near the BCM binding pocket and are also adjacent to the ATP and secondary RNA binding sites.

In Rho functional assays, the three mutants Ser264Cys, Ser266Ala, and Leu208Arg were found to be highly resistant to $B C M$ inhibition, suggesting that these residues may directly interact with BCM (Magyar et al., 
A
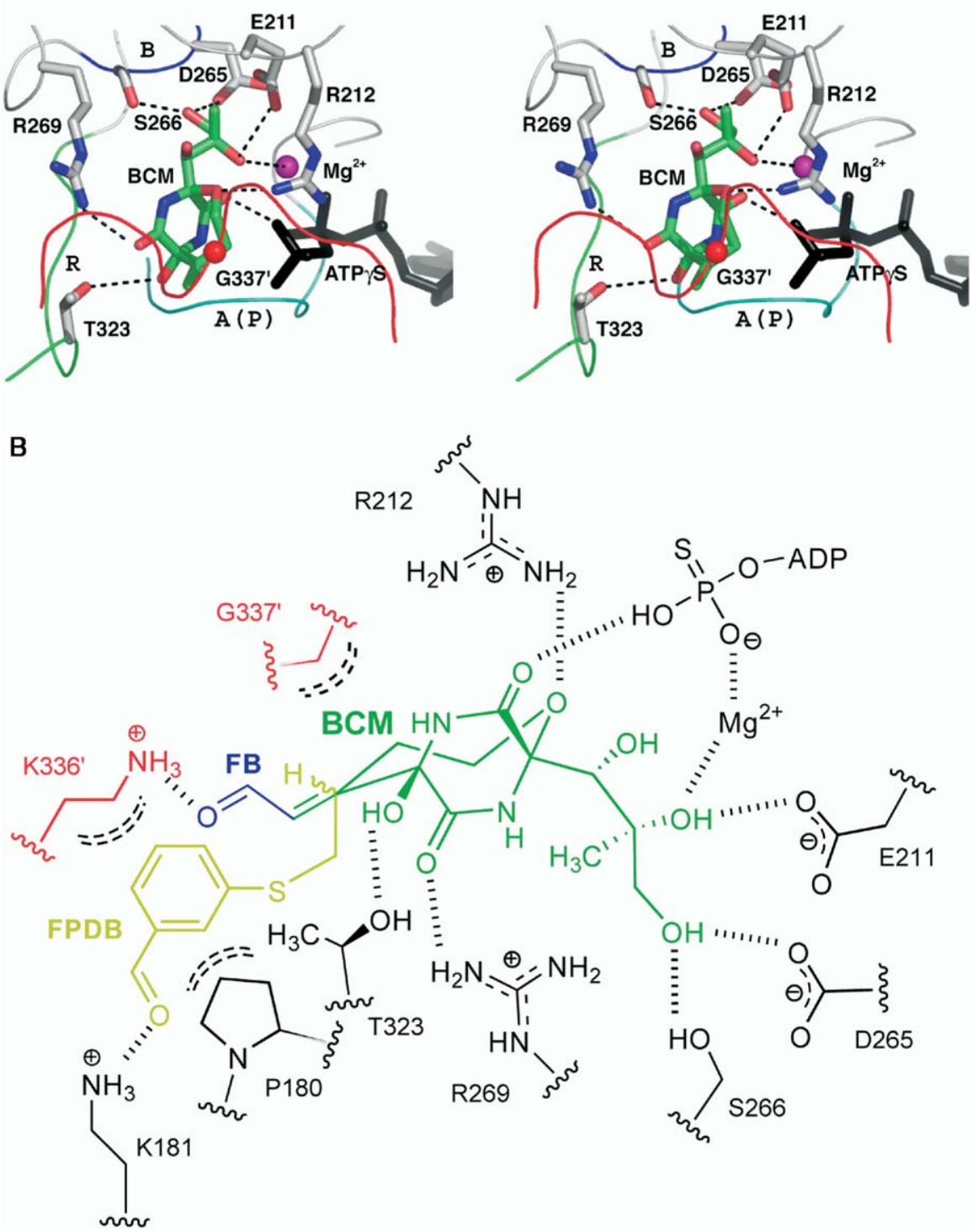

Figure 4. Rho-BCM Contacts

(A) Stereo view of BCM (colored stick) bound to Rho. Amino acids involved in coordinating BCM are shown as gray sticks (Table 3 ). Mg ${ }^{2+}$ and ATP $\gamma \mathrm{S}$ are shown as a magenta sphere and as black sticks.

(B) Schematic of Rho-BCM contacts. Protein residues are colored black. BCM is shown in green. The additional contacts between the protein and the aldehyde groups of FPDB (yellow) and FB (blue) at the $\mathrm{C}(5 \mathrm{a})$-position of the [4.2.2] piperazinedione ring are also shown.

1999; Moyse et al., 2000, 2001; Zwiefka et al., 1993). In the Rho•BCM complex, the side chain of Ser266 lines the interior of the BCM binding pocket and makes a hydrogen bond with the $\mathrm{C}\left(3^{\prime}\right) \mathrm{OH}$ of the $\mathrm{C}(1)$ triol moiety (Figures 4 and 5A). Loss of the serine hydroxyl by substitution with cysteine would abolish this interaction, decreasing the affinity of the BCM binding pocket for the inhibitor. By contrast, Leu208 lies $6.5 \AA$ away from $B C M$ and does not interact directly with the antibiotic (Figure 5A). This amino acid is, however, part of the hydrophobic core of the ATP binding domain and is located adjacent to Glu211 and Arg212, two residues that directly contact BCM (Figure 4). Introduction of a large hydrophilic side chain in this location would be expected to reposition helix $\alpha 8$ and its associated connector loop, thereby perturbing the orientation of Glu211 and Arg212 and remodeling two key binding determinants of the BCM binding pocket.

A second set of BCM-resistant mutations, Met219Lys and Gly337Ser, have also been reported (Magyar et al., 1999; Zwiefka et al., 1993). These substitutions are less resistant to BCM than are changes at Ser266 and Leu208. Significantly, the Met219Lys and Gly337Ser mutants increase the affinity of Rho for ssRNA, suggesting that their mild resistance to BCM could result from a diminished capacity of the drug to displace RNA 


\begin{tabular}{|c|c|c|c|}
\hline Bicyclomycin/Atom & Rho/Residue & Rho/Atom & Distance \\
\hline $\mathrm{O} 2$ & Arg212 & $\mathrm{N} \in 1$ & 2.9 \\
\hline $\mathrm{OH}\left(2^{\prime}\right)$ & $\mathrm{Mg}^{2+}$ & $\mathrm{Mg}^{2+}$ & 2.8 \\
\hline $\mathrm{OH}\left(2^{\prime}\right)$ & Glu211 & $\mathrm{O} \in 2$ & 2.9 \\
\hline $\mathrm{OH}\left(3^{\prime}\right)$ & Asp265 & O $\delta 1$ & 2.6 \\
\hline $\mathrm{OH}\left(3^{\prime}\right)$ & Ser266 & $\mathrm{O} \gamma$ & 2.4 \\
\hline $\mathrm{C}(7) \mathrm{O}$ & Arg269 & $\mathrm{N} \in 2$ & 3.2 \\
\hline $\mathrm{C}(6) \mathrm{OH}$ & Thr323 & Or1 & 3.4 \\
\hline $\mathrm{C}(9) \mathrm{O}$ & ATP $\gamma \mathrm{S}$ & $\mathrm{OH}$ & 2.7 \\
\hline $\mathrm{C} 3, \mathrm{C} 4$ & Gly337' & $\mathrm{C} \alpha$ & 3.0 \\
\hline \multicolumn{4}{|l|}{ FPDB } \\
\hline Thiobenzaldehyde & Lys181 & $\mathrm{N} \zeta$ & 3.4 \\
\hline Thiobenzaldehyde & Lys336' & aliphatic side chain & 4.4 \\
\hline Thiobenzaldehyde & Pro180 & aliphatic ring & 4.2 \\
\hline \multicolumn{4}{|l|}{ FB } \\
\hline Formyl & Lys336' & $\mathrm{N} \zeta$ & 2.7 \\
\hline
\end{tabular}

from the secondary RNA binding sites (Magyar et al., $1999,1996)$. Inspection of the Rho BCM structure reveals that Met219 resides a considerable distance away from the BCM binding pocket $(\sim 15 \AA)$ and that its side chain forms part of the hydrophobic core of the C-terminal domain (Figure 5). Although Met219 lies near the secondary RNA binding site, it is not accessible for interactions with RNA. As a consequence, the increase in RNA binding affinity observed for this mutant most likely arises from a conformational rearrangement in this region. With respect to its effect on BCM resistance, Met219 extends from strand $\beta 7$ of the connector loop that carries Glu211 and Arg212. As with Leu208, substitution of a large charged side chain for Met219 would presumably reposition strand $\beta 7$ and indirectly perturb the ability of the Glu211 carboxylate to associate with the $\mathrm{BCM} C(1)$ triol group.

By contrast to Met219, Gly337' is an integral part of one wall of the BCM binding pocket. This amino acid is located on helix $\alpha 12^{\prime}$, which sits right above the $R$ loop (Figure 2B). The Rho•BCM structure shows that Gly337' makes van der Waals contacts $(2.8 \AA)$ to the ether group of the [4.2.2] piperazinedione ring (Figures 4 and $5 \mathrm{~A}$ ). The side chain of a residue larger than Gly337', such as serine, would extend into the BCM binding pocket and impede BCM binding. Furthermore, Gly337' is adjacent to Lys336', a residue in the secondary RNA binding site that has been suggested to contact RNA directly. Substitution of glycine with serine would add an additional hydrogen-bonding group to this region and could account for the enhancement of RNA binding to the secondary site.

\section{Chemical Modifications of BCM}

Prior to our structural studies, numerous attempts have been made to improve the inhibitory properties of $\mathrm{BCM}$ by chemically modifying the antibiotic (Park $\mathrm{Hg}$ et al., 1996a, 1996b; Santillan et al., 1996, 1998; Vincent et al., 2001). Most changes to BCM resulted in either partial or complete loss of inhibitory activity. For example, modification of the $\mathrm{C}(1)$ triol group at the $\mathrm{C}\left(1^{\prime}\right), \mathrm{C}\left(2^{\prime}\right)$, or $\mathrm{C}\left(3^{\prime}\right)$ positions all produced compounds with significantly poorer inhibition profiles than seen for BCM (Park Hg et al., 1996a). Our work accounts for this ob- servation, showing that the $\mathrm{C}(1)$ triol group fits snugly into the BCM binding pocket, where it is extensively coordinated by a network of hydrogen bonds to specific residues of Rho (Figure 4).

Attempts to chemically modify the [4.2.2] piperazinedione bicyclic unit to improve BCM activity have also been unsuccessful. For example, methyl substitution at either amide nitrogen within the bicyclic unit or substitution of the hydroxyl at the $\mathrm{C}(6)$ position with either alkoxy, thioalkoxy, thiophenoxy, anilino, or hydrogen groups completely abrogated their inhibitory activity (Santillan et al., 1996). In all three Rho-inhibitor crystal structures, the bicyclic unit is sandwiched between the side chain of Arg269 and the oxygens of the $\gamma$-phosphate of bound nucleotide. Most modifications of the [4.2.2] bicyclic unit would therefore appear to either sterically block binding or would abrogate hydrogen bonds and van der Waals contacts that stabilize binding. Notably, the rigid bicyclic ring of BCM adopts a "twist boat" conformation (Park Hg et al., 1996b; Santillan et al., 1996), which our structural study clearly shows favors interactions with residues that line the interior of the BCM binding pocket. In particular, substitution of the bicyclic unit with a nearly planar ring system may eliminate binding interactions with the amide carbonyls of the [4.2.2] piperazinedione ring system, which make a number of key contacts to the protein.

Substitution of the $C(6)$ hydroxyl with alkoxy and thioalkoxy substituents also have been shown to decrease BCM binding (Santillan et al., 1998). In our structure, the $\mathrm{C}(6)$ hydroxyl extends toward the $\mathrm{R}$ loop and forms a weak hydrogen bond with Thr323 ( $3.4 \AA$ A). The introduction of a larger substituent at this position would interfere sterically with the R loop, while introduction of a smaller substituent would abolish this interaction. Either event would account for the reduction in binding affinity of the inhibitor for Rho.

The only BCM modifications that have thus far increased inhibitory activity are those at the $\mathrm{C}(5)-\mathrm{C}(5 \mathrm{a})$ double bond (Figure 1) (Brogan et al., 2003; Park Hg et al., 1996b; Vincent et al., 2001). For example, select $\mathrm{C}(5)$-unsaturated and $\mathrm{C}(5 \mathrm{a})$-substituted $\mathrm{C}(5)-\mathrm{C}(5 \mathrm{a})$-dihydrobicyclomycins exhibited comparable or better inhibitory activity compared with BCM. Our structural data show that the $C(5)-C(5 a)$ exomethylene moiety points 

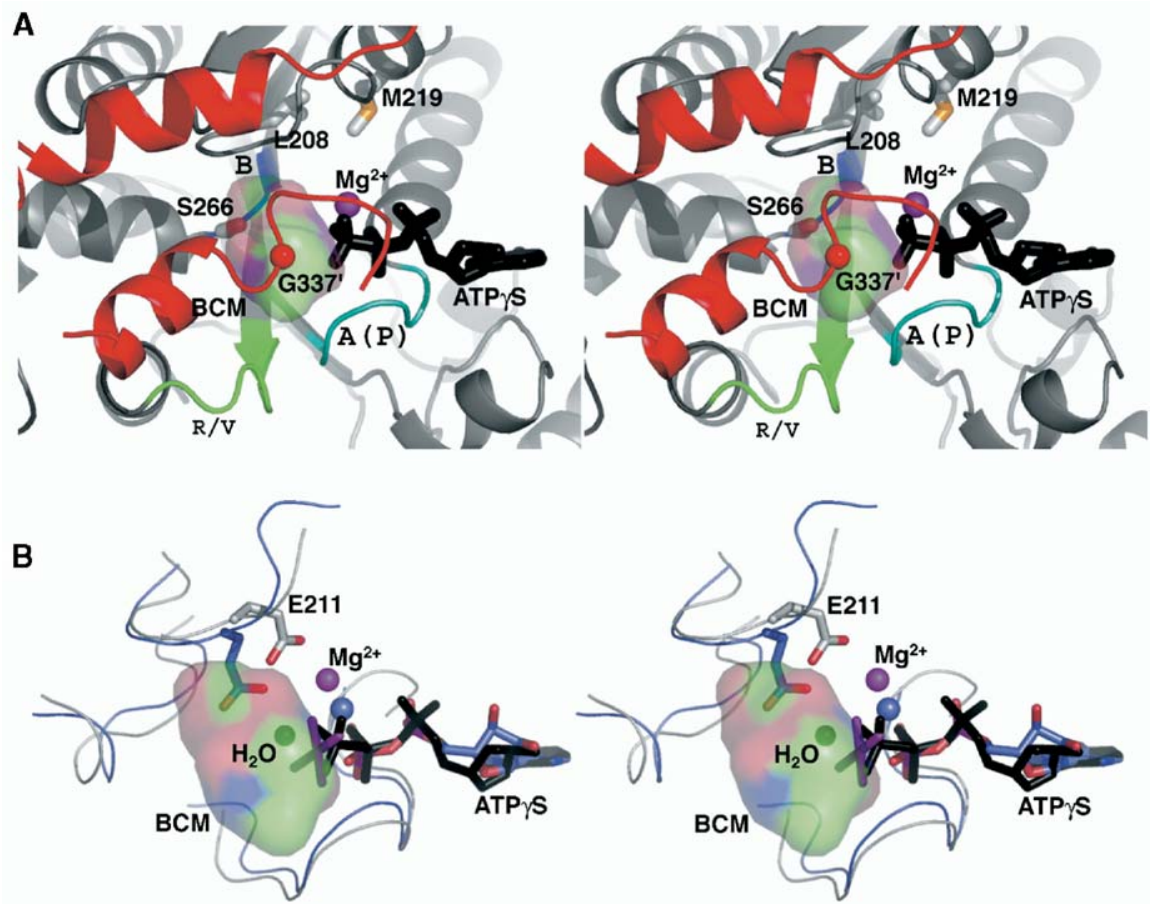

Figure 5. BCM-Resistance Mutations and Mechanism of ATPase Inhibition

(A) Stereo view of the dimer interface rotated $90^{\circ}$ from that in Figure $2 \mathrm{~B}$, showing the location of BCM-resistant mutants of Rho as sticks. Protomer $\mathrm{C}$ is colored red and protomer $\mathrm{D}$ is gray. The key signature motifs important for RNA (R loop) and ATP (Walker-A and -B) binding are highlighted in green, cyan, and blue, respectively.

(B) Stereo view of the $F_{1}$-ATPase (slate, PDB model $1 \mathrm{H} 8 \mathrm{E}$ ) active site superposed on the Rho•BCM complex (gray). BCM (transparent surface) occupies the same space as the hydrolytic water molecule (black sphere) seen coordinating the AlF $F_{4}$ moiety in the $F_{1}$-ATPase. The nucleotides and $\mathrm{Mg}^{2+}$ ions in both structures appear to be organized similarly, with the exception of the $\gamma$-phosphate, which is offset slightly by BCM binding.

away from the BCM binding pocket toward an open cavity and does not interact with the protein. Thus, the introduction of larger chemical groups at this position is poised to foster additional contacts with residues that lie in proximity to the $\mathrm{C}(5)-\mathrm{C}(5 \mathrm{a})$ end of $\mathrm{BCM}$. In the case of FPDB, our structural studies show that the extra thiobenzaldehyde group extends from the BCM binding pocket to make van der Waals contacts with Pro180 of the same protomer and the aliphatic portion of the side chain of Lys336' on the adjacent protomer. The terminal carbonyl of this moiety also appears capable of hydrogen bonding to Lys181, although the electron density for this element is somewhat poor, suggesting that the thiobenzaldehyde group is mobile and may make other contacts with the protein. Together, these new interactions account for the 15-fold increase in inhibitory activity of FPDB compared with $B C M$. By contrast, the inhibitory activity of FB is similar to that of BCM (Vincent et al., 2000b). This observation likewise appears consistent with our structural data, since only one protein $\bullet$ inhibitor contact is observed for the $\mathrm{C}(5)-\mathrm{C}(5 \mathrm{a})$ modification of FB (between FB's formyl group and Lys336' of the C-terminal domain adjacent to the primary BCM binding site), as opposed to the three contacts seen between FPDB and Rho. In addition, the geometry of the Lys336' $\bullet$ formyl hydrogen bond appears somewhat strained and could offset the energetic benefits of FB's additional interaction with Rho, although the resolution of our data precludes a definitive assessment of this observation.

\section{Mechanism of Inhibition}

Prior in vitro studies have shown that the addition of BCM leads to the production of unusually long transcripts (Magyar et al., 1996; Zwiefka et al., 1993). Homology modeling studies using the $\mathrm{F}_{1}$-ATPase previously indicated that BCM-resistance mutations cluster near the secondary RNA tracking site and the ATP binding pocket (Magyar et al., 1996; Park et al., 1995; Riba et al., 1998; Vincent et al., 2000a, 2001, 2000b). Kinetic ATPase assays also showed that BCM is a noncompetitive inhibitor that does not compete directly for ATP binding (Park et al., 1995), and whose target site may partially overlap with the secondary RNA binding site in Rho (Carrano et al., 1998; Magyar et al., 1999, 1996; Moyse et al., 2000, 2001). These observations suggested that BCM associates with a nucleotide-bound state of Rho in a manner that diminishes ATP turnover and RNA binding to disrupt translocation and corrupt Rho-dependent transcription termination in vivo (Magyar et al., 1996; Zwiefka et al., 1993).

Given that BCM and ATP binding are independent of each other, how do bicyclomycins decrease Rho's ATPase activity? Several means are directly evident 
from the Rho ${ }^{B C M}$ crystal structure. For example, the $C(9)$ amide carbonyl group within the [4.2.2] bicyclic subunit of BCM contacts the $\gamma$-phosphate of bound nucleotide (Figure 4). This interaction could hinder either ATP hydrolysis and/or phosphate release. Another clue to the mechanism of BCM inhibition comes from consideration of the ATP hydrolysis reaction carried out by RecA-type ATPases. Although the Rho protein imaged here does not adopt a conformation competent to hydrolyze ATP, principally because of the widened gap between subunits in the open-ring state, we can model this configuration using the homologous closed-ring $F_{1}$-ATPase structure (Figure 5B) (Abrahams et al., 1994). In the $F_{1}$-ATPase, the water molecule that participates in nucleophilic attack on ATP is coordinated by a glutamic acid that lies proximal to the Walker-A and -B signature sequence motifs (Weber et al., 2000). Given that the ATPase domains of $F_{1}$ and Rho are $\sim 40 \%$ identical, a similar arrangement has been predicted to occur for Glu211 of Rho (Weber et al., 2003). Surpris-

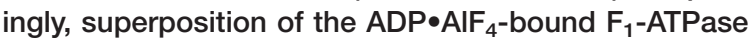
structure (1H8E) (Braig et al., 2000) on our Rho•BCM complex shows that $\mathrm{BCM}$ physically occludes the site normally occupied by the attacking water molecule. $\mathrm{BCM}$ also subtly repositions the $\gamma$-phosphate $\sim 5^{\circ}$ away from the location it normally occupies prior to hydrolysis. These effects, which to our knowledge have not been seen in other ATPase-inhibitor complexes, completely account for the ability of BCM to act as a noncompetitive inhibitor of ATP turnover. Moreover, because part of the BCM binding site is formed by the $R$ loop, a region required for mRNA translocation, our data show that $\mathrm{BCM}$ may be capable of perturbing RNA associations as well. This finding is consistent with the mixed type of inhibition previously observed for BCM in Rho functional assays, using short oligoribonucleotides that bind to the secondary site (Magyar et al., 1996).

In summary, our structural studies provide a clear picture of the mechanism by which BCM, a compound in current use, inhibits the transcription termination factor Rho. These data also represent a structural example of an unusual mode of noncompetitive inhibition and a rare view of a nonnucleotide inhibitor bound to a hexameric helicase/translocase. Taken together, our observations reveal the molecular basis by which BCM perturbs Rho function to disrupt gene regulation in vivo and promote bacterial cell death.

\section{Experimental Procedures}

Protein Expression, Purification, and Crystallization The full-length $E$. coli Rho protein was expressed and purified using the procedure described previously (Skordalakes and Berger, 2003). Rho•nucleotide $\bullet R N A \bullet i n h i b i t o r$ cocrystals were grown by vapor diffusion at room temperature. Prior to crystallization, Rho was mixed with the single-stranded RNA substrate $\mathrm{r}(\mathrm{CU})_{4}$, dialyzed against $10 \mathrm{mM}$ Tris- $\mathrm{HCl}\left(\mathrm{pH} \mathrm{7.5)}\right.$ and $50 \mathrm{mM} \mathrm{NaCl}$ at $4^{\circ} \mathrm{C}$, and then mixed with $5 \mathrm{mM}$ ATP $\gamma \mathrm{S}, 10 \mathrm{mM} \mathrm{MgCl}$ and a 3- to 5-fold molar excess of inhibitor. For BCM and FPDB, one volume of this stock solution was mixed with an equal volume of crystallization solution containing $100 \mathrm{mM} \mathrm{Na} \cdot$ Cacodylate (pH 6.5), $100 \mathrm{mM} \mathrm{NaCl}, 8 \%$ PEG $8 \mathrm{~K}, 40 \%$ glycerol, $0.15 \mathrm{mM}$ FOS-Choline-12; the well solution was then diluted with an equivalent amount of $\mathrm{H}_{2} \mathrm{O}$ prior to sealing the drop over the reservoir. Small crystals appeared overnight and grew to average dimensions of $50 \times 150 \times 150 \mu \mathrm{M}$ in 2 weeks.
Cocrystallization with FB proceeded similarly, except that crystals grew from a solution containing $100 \mathrm{mM} \mathrm{Na} \bullet$ Cacodylate $(\mathrm{pH} 6.5)$, $100 \mathrm{mM} \mathrm{NaCl}, 100 \mathrm{mM} \mathrm{Na}_{2} \mathrm{HPO}_{4}, 10 \%$ PEG 3350, 30\% glycerol. Small crystals appeared in 3-5 days and grew to average dimensions of $30 \times 30 \times 200 \mu \mathrm{M}$ in 2 weeks. Rho crystals grown in the presence of BCM and FPDB were harvested directly into cryoprotectant consisting of $50 \mathrm{mM} \mathrm{Na} \cdot$ Cacodylate $(\mathrm{pH} 6.5), 50 \mathrm{mM} \mathrm{NaCl}$, 5\% PEG 8K, 0.15 mM FOS-Choline-12, 20\% glycerol, 5\% PEG 400 and drug, then flash frozen in liquid nitrogen prior to data collection, while Rho• FB cocrystals were harvested into $50 \mathrm{mM} \mathrm{Na} \cdot \mathrm{Ca}-$ codylate (pH 6.5), $50 \mathrm{mM} \mathrm{NaCl}, 50 \mathrm{mM} \mathrm{Na} \mathrm{HPO}_{4}$ 6\% PEG 3350, $15 \%$ glycerol, and $10 \%$ PEG 400 plus drug before flash freezing. Cell dimensions and space group information for all cocrystals are given in Table 1.

\section{Data Collection, Structure Determination, and Refinement}

Data were collected at beamline 8.3.1 at the Advanced Light Source and processed with Elves (Holton and Alber, 2004) (Table 1). Phases for all three Rho-bicyclomycin complexes were obtained by molecular replacement (AmoRe [Navaza, 2001]) using the inhibitor-free and AMPPNP-free Rho hexamer as a model (1PV4). Coordinates for the antibiotics were generated using PRODRG (van Aalten et al., 1996) and built into $F_{o}-F_{c}$ and multidomain-averaged electron density maps. All model building was carried out using $\mathrm{O}$ (Jones and Kjeldgaard, 1997), and structures were refined with REFMAC5 (Murshudov et al., 1997). Simulated-annealing omit maps (CNS [Brünger et al., 1998]) were used to further validate the placement of the inhibitor. The final $\mathbf{R}_{\text {work }}, \mathbf{R}_{\text {free }}$, and Ramachandran statistics for all models are given in Table 2. All molecular figures were generated using PYMOL (DeLano, 2002).

Supplemental Data

Supplemental Data are available at http://www.structure.org/cgi/ content/full/13/1/99/DC1/.

\section{Acknowledgments}

We thank Dr. Y. Itoh and the Fujisawa Pharmaceutical Co., Ltd., Japan, for the gift of BCM. This work was supported by the $\mathrm{Na}$ tional Institutes of Health grant GM37934 (H.K.) and by generous funding from the G. Harold and Leila Y. Mathers Charitable Foundation. The American Chemical Society Division of Medicinal Chemistry and Bristol-Myers Squibb are gratefully acknowledged for support of a predoctoral fellowship to A.P.B.

Received: September 10, 2004

Revised: October 20, 2004

Accepted: October 20, 2004

Published: January 11, 2005

\section{References}

Abrahams, J.P., Leslie, A.G., Lutter, R., and Walker, J.E. (1994). Structure at 2.8 A resolution of F1-ATPase from bovine heart mitochondria. Nature 370, 621-628.

Alifano, P., Rivellini, F., Limauro, D., Bruni, C.B., and Carlomagno, M.S. (1991). A consensus motif common to all Rho-dependent prokaryotic transcription terminators. Cell 64, 553-563.

Allison, T.J., Wood, T.C., Briercheck, D.M., Rastinejad, F., Richardson, J.P., and Rule, G.S. (1998). Crystal structure of the RNA-binding domain from transcription termination factor rho. Nat. Struct. Biol. 5, 352-356.

Braig, K., Menz, R.I., Montgomery, M.G., Leslie, A.G., and Walker, J.E. (2000). Structure of bovine mitochondrial F(1)-ATPase inhibited by $\mathrm{Mg}(2+) \mathrm{ADP}$ and aluminium fluoride. Struct. Fold. Des. 8, 567573

Brennan, C.A., Dombroski, A.J., and Platt, T. (1987). Transcription termination factor rho is an RNA-DNA helicase. Cell 48, 945-952.

Brogan, A.P., Widger, W.R., and Kohn, H. (2003). Bicyclomycin fluo- 
rescent probes: synthesis and biochemical, biophysical, and biological properties. J. Org. Chem. 68, 5575-5587.

Brünger, A., Adams, P., Clore, G., DeLano, W., Gros, P., GrosseKunstleve, R., Jiang, J.-S., Kuszewski, J., Nilges, M., Pannu, N., et al. (1998). Crystallography \& NMR system: a new software suite for macromolecular structure determination. Acta Crystallogr. D Biol. Crystallogr. 54, 905-921.

Burgess, B.R., and Richardson, J.P. (2001). RNA passes through the hole of the protein hexamer in the complex with the Escherichia coli Rho factor. J. Biol. Chem. 276, 4182-4189.

Carrano, L., Bucci, C., De Pascalis, R., Lavitola, A., Manna, F., Corti, E., Bruni, C.B., and Alifano, P. (1998). Effects of bicyclomycin on RNA- and ATP-binding activities of transcription termination factor Rho. Antimicrob. Agents Chemother. 42, 571-578.

Cavarelli, J., Rees, B., Ruff, M., Thierry, J.C., and Moras, D. (1993). Yeast tRNA(Asp) recognition by its cognate class II aminoacyltRNA synthetase. Nature 362, 181-184.

Conway, A.B., Lynch, T.W., Zhang, Y., Fortin, G.S., Fung, C.W., Symington, L.S., and Rice, P.A. (2004). Crystal structure of a Rad51 filament. Nat. Struct. Mol. Biol. 11, 791-796.

DeLano, W.L. (2002). The PyMOL Molecular Graphics System (San Carlos, CA: DeLano Scientific).

Engel, D., and Richardson, J.P. (1984). Conformational alterations of transcription termination protein rho induced by ATP and by RNA. Nucleic Acids Res. 12, 7389-7400.

Ericsson, C.D., DuPont, H.L., Galindo, E., Mathewson, J.J., Morgan, D.R., Wood, L.V., and Mendiola, J. (1985). Efficacy of bicozamycin in preventing traveler's diarrhea. Gastroenterology 88, 473-477.

Gogol, E.P., Seifried, S.E., and von Hippel, P.H. (1991). Structure and assembly of the Escherichia coli transcription termination factor rho and its interaction with RNA. I. Cryoelectron microscopic studies. J. Mol. Biol. 221, 1127-1138.

Gorbalenya, A.E., Koonin, E.V., Donchenko, A.P., and Blinov, V.M. (1989). Two related superfamilies of putative helicases involved in replication, recombination, repair and expression of DNA and RNA genomes. Nucleic Acids Res. 17, 4713-4730.

Hall, M.C., and Matson, S.W. (1999). Helicase motifs: the engine that powers DNA unwinding. Mol. Microbiol. 34, 867-877.

Holton, J., and Alber, T. (2004). Automated protein crystal structure determination using ELVES. Proc. Natl. Acad. Sci. USA 101, 15371542.

Iseki, M., Miyoshi, T., Konomi, T., and Imanaka, H. (1980). Biosynthesis of bicyclomycin. II. Biosynthetic conditions and incorporation of radioactive precursors into bicyclomycin by washed mycelium. J. Antibiot. (Tokyo) 33, 488-493.

Jones, T.A., and Kjeldgaard, M. (1997). O: The Manual, Ver. 5.10 (Sweden: Uppsala University).

Kamiya, T., Maeno, S., Hashimoto, M., and Mine, Y. (1972). Bicyclomycin, a new antibiotic. II. Structural elucidation and acyl derivatives. J. Antibiot. (Tokyo) 25, 576-581.

Kim, D.E., and Patel, S.S. (2001). The kinetic pathway of RNA binding to the Escherichia coli transcription termination factor Rho. J. Biol. Chem. 276, 13902-13910.

Kim, D.E., Shigesada, K., and Patel, S.S. (1999). Transcription termination factor Rho contains three noncatalytic nucleotide binding sites. J. Biol. Chem. 274, 11623-11628.

Lowery-Goldhammer, C., and Richardson, J.P. (1974). An RNAdependent nucleoside triphosphate phosphohydrolase (ATPase) associated with rho termination factor. Proc. Natl. Acad. Sci. USA 71, 2003-2007.

Magyar, A., Zhang, X., Kohn, H., and Widger, W.R. (1996). The antibiotic bicyclomycin affects the secondary RNA binding site of Escherichia coli transcription termination factor Rho. J. Biol. Chem. 271, 25369-25374.

Magyar, A., Zhang, X., Abdi, F., Kohn, H., and Widger, W.R. (1999). Identifying the bicyclomycin binding domain through biochemical analysis of antibiotic-resistant rho proteins. J. Biol. Chem. 274, 7316-7324.

Martinez, A., Burns, C.M., and Richardson, J.P. (1996a). Residues in the RNP1-like sequence motif of Rho protein are involved in RNAbinding affinity and discrimination. J. Mol. Biol. 257, 909-918.

Martinez, A., Opperman, T., and Richardson, J.P. (1996b). Mutational analysis and secondary structure model of the RNP1-like sequence motif of transcription termination factor Rho. J. Mol. Biol. 257, 895-908.

Miwa, Y., Horiguchi, T., and Shigesada, K. (1995). Structural and functional dissections of transcription termination factor rho by random mutagenesis. J. Mol. Biol. 254, 815-837.

Miyamura, S., Ogasawara, N., Otsuka, H., Niwayama, S., and Tanaka, H. (1972). Antibiotic no. 5879, a new water-soluble antibiotic against gram-negative bacteria. J. Antibiot. (Tokyo) 25, 610-612.

Miyamura, S., Ogasawara, N., Otsuka, H., Niwayama, S., and Tanaka, H. (1973). Antibiotic 5879 produced by Streptomyces aizunensis, identical with bicyclomycin. J. Antibiot. (Tokyo) 26, 479-484.

Miyoshi, T., Iseki, M., Konomi, T., and Imanaka, H. (1980). Biosynthesis of bicyclomycin. I. Appearance of aerial mycelia negative strains (am-). J. Antibiot. (Tokyo) 33, 480-487.

Morgan, W.D., Bear, D.G., Litchman, B.L., and von Hippel, P.H. (1985). RNA sequence and secondary structure requirements for rho-dependent transcription termination. Nucleic Acids Res. 13, 3739-3754.

Moyse, K.A., Knight, J.S., and Richardson, J.P. (2000). The bicyclomycin sensitivities of 38 bicyclomycin-resistant mutants of transcription termination protein rho and the location of their mutations support a structural model of rho based on the F(1) ATPase. J. Mol. Biol. 302, 565-579.

Moyse, K.A., Knight, J.S., and Richardson, J.P. (2001). Phenotypic characterization of a comprehensive set of bicyclomycin-resistant mutants. Biochim. Biophys. Acta 1520, 223-233.

Murshudov, G.N., Vagin, A.A., and Dodson, E.J. (1997). Refinement of macromolecular structures by the maximum-likelihood method. Acta Crystallogr. D Biol. Crystallogr. 53, 240-255.

Navaza, J. (2001). Implementation of molecular replacement in AMoRe. Acta Crystallogr. D Biol. Crystallogr. 57, 1367-1372.

Nowatzke, W.L., Keller, E., Koch, G., and Richardson, J.P. (1997). Transcription termination factor Rho is essential for Micrococcus luteus. J. Bacteriol. 179, 5238-5240.

Oda, T., and Takanami, M. (1972). Observations on the structure of the termination factor rho and its attachment to DNA. J. Mol. Biol. 71, 799-802.

Park, H.G., Zhang, X., Moon, H.S., Zwiefka, A., Cox, K., Gaskell, S.J., Widger, W.R., and Kohn, H. (1995). Bicyclomycin and dihydrobicyclomycin inhibition kinetics of Escherichia coli rho-dependent transcription termination factor ATPase activity. Arch. Biochem. Biophys. 323, 447-454.

Park Hg, H., Zhang, X., Widger, W.R., and Kohn, H. (1996a). Role of the $\mathrm{C}(1)$ triol group in bicyclomycin: synthesis and biochemical and biological properties. J. Org. Chem. 61, 7750-7755.

Park Hg, H., Zhang, Z., Zhang, X., Widger, W.R., and Kohn, H. (1996b). Role of the $\mathrm{C}(5)-\mathrm{C}(5 \mathrm{a})$ exomethylene group in bicyclomycin: synthesis, structure, and biochemical and biological properties. J. Org. Chem. 61, 7764-7776.

Patel, S.S., and Picha, K.M. (2000). Structure and function of hexameric helicases. Annu. Rev. Biochem. 69, 651-697.

Riba, I., Gaskell, S.J., Cho, H., Widger, W.R., and Kohn, H. (1998). Evidence for the location of bicyclomycin binding to the Escherichia coli transcription termination factor Rho. J. Biol. Chem. 273, 34033-34041.

Richardson, J. (2002). Rho-dependent termination and ATPases in transcript termination. Biochim. Biophys. Acta 1577, 251-260.

Santillan, A., Jr., Park Hg, H., Zhang, X., Lee, O.S., Widger, W.R., and Kohn, H. (1996). Role of the [4.2.2] Bicyclic Unit in Bicyclomycin: Synthesis, Structure, Chemical, Biochemical, and Biological Properties. J. Org. Chem. 61, 7756-7763.

Santillan, A., Jr., Zhang, X., Hardesty, J., Widger, W.R., and Kohn, H. (1998). Role of the C(6)-hydroxy group in bicyclomycin: synthesis, structure, and chemical, biochemical, and biological properties. J. Med. Chem. 41, 1185-1194. 
Sawaya, M.R., Guo, S., Tabor, S., Richardson, C.C., and Ellenberger, T. (1999). Crystal structure of the helicase domain from the replicative helicase-primase of bacteriophage T7. Cell 99, 167-177.

Schindelin, H., Jiang, W., Inouye, M., and Heinemann, U. (1994). Crystal structure of CspA, the major cold shock protein of Escherichia coli. Proc. Natl. Acad. Sci. USA 91, 5119-5123.

Shigesada, K., and Wu, C.W. (1980). Studies of RNA release reaction catalyzed by $\mathrm{E}$. coli transcription termination factor rho using isolated ternary transcription complexes. Nucleic Acids Res. 8, 3355-3369.

Singleton, M.R., Sawaya, M.R., Ellenberger, T., and Wigley, D.B. (2000). Crystal structure of T7 gene 4 ring helicase indicates a mechanism for sequential hydrolysis of nucleotides. Cell 101, 589-600.

Skordalakes, E., and Berger, J.M. (2003). Structure of the Rho transcription terminator: mechanism of mRNA recognition and helicase loading. Cell 114, 135-146.

Stitt, B.L. (2001). Escherichia coli transcription termination factor Rho binds and hydrolyzes ATP using a single class of three sites. Biochemistry 40, 2276-2281.

Story, R.M., Weber, I.T., and Steitz, T.A. (1992). The structure of the E. coli recA protein monomer and polymer. Nature 355, 318-325.

Toth, E.A., Li, Y., Sawaya, M.R., Cheng, Y., and Ellenberger, T. (2003). The crystal structure of the bifunctional primase-helicase of bacteriophage T7. Mol. Cell 12, 1113-1123.

Uemura, R., Sueyoshi, M., Nagayoshi, M., and Nagatomo, H. (2003). Antimicrobial susceptibilities of Shiga toxin-producing Escherichia coli isolates from pigs with edema disease in Japan. Microbiol. Immunol. 47, 57-61.

van Aalten, D.M., Bywater, R., Findlay, J.B., Hendlich, M., Hooft, R.W., and Vriend, G. (1996). PRODRG, a program for generating molecular topologies and unique molecular descriptors from coordinates of small molecules. J. Comput. Aided Mol. Des. 10, 255262.

VanLoock, M.S., Yu, X., Yang, S., Lai, A.L., Low, C., Campbell, M.J., and Egelman, E.H. (2003). ATP-mediated conformational changes in the RecA filament. Structure (Camb.) 11, 187-196.

Vincent, F., Openshaw, M., Trautwein, M., Gaskell, S.J., Kohn, H., and Widger, W.R. (2000a). Rho transcription factor: symmetry and binding of bicyclomycin. Biochemistry 39, 9077-9083.

Vincent, F., Widger, W.R., Openshaw, M., Gaskell, S.J., and Kohn, H. (2000b). 5a-formylbicyclomycin: studies on the bicyclomycin-Rho interaction. Biochemistry 39, 9067-9076.

Vincent, F., Srinivasan, J., Santillan, A., Jr., Widger, W.R., and Kohn, H. (2001). C(5)-C(5a)-modified bicyclomycins: synthesis, structure, and biochemical and biological properties. J. Org. Chem. 66, 2251-2264.

Walstrom, K.M., Dozono, J.M., Robic, S., and von Hippel, P.H. (1997). Kinetics of the RNA-DNA helicase activity of Escherichia coli transcription termination factor rho. 1. Characterization and analysis of the reaction. Biochemistry 36, 7980-7992.

Weber, J., Nadanaciva, S., and Senior, A.E. (2000). ATP-driven rotation of the gamma subunit in F(1)-ATPase. FEBS Lett. 483, 1-5.

Weber, T.P., Widger, W.R., and Kohn, H. (2003). Metal dependency for transcription factor rho activation. Biochemistry 42, 1652-1659.

Wu, Y., He, Y., Moya, I.A., Qian, X., and Luo, Y. (2004). Crystal structure of archaeal recombinase RADA: a snapshot of its extended conformation. Mol. Cell 15, 423-435.

Xu, Y., Kohn, H., and Widger, W.R. (2002). Mutations in the rho transcription termination factor that affect RNA tracking. J. Biol. Chem. 277, 30023-30030.

Xu, Y., Johnson, J., Kohn, H., and Widger, W.R. (2003). ATP binding to Rho transcription termination factor. Mutant F355W ATP-induced fluorescence quenching reveals dynamic ATP binding. J. Biol. Chem. 278, 13719-13727.

Yu, X., Horiguchi, T., Shigesada, K., and Egelman, E.H. (2000). Three-dimensional reconstruction of transcription termination factor rho: orientation of the N-terminal domain and visualization of an RNA-binding site. J. Mol. Biol. 299, 1279-1287.
Zhu, A.Q., and von Hippel, P.H. (1998). Rho-dependent termination within the trp $t^{\prime}$ terminator. I. Effects of rho loading and template sequence. Biochemistry 37, 11202-11214.

Zwiefka, A., Kohn, H., and Widger, W.R. (1993). Transcription termination factor rho: the site of bicyclomycin inhibition in Escherichia coli. Biochemistry 32, 3564-3570.

\section{Accession Numbers}

The Rho-BCM, Rho-FPDB, and Rho-FB coordinates have been deposited into the RCSB Protein Data Bank under the accession numbers 1XPO, 1XPU, and 1XPR, respectively. 